\title{
Training in Micro-Vascular Anastomosis Using Rat Femoral Vessels: Comparison of Immediate and Delayed Patency Rates
}

\author{
Nupur PRUTHI ${ }^{1}$, Pragyan SARMA ${ }^{1}$, Paritosh PANDEY² \\ ${ }^{1}$ National Institute of Mental Health and Neurosciences, Department of Neurosurgery, Bangalore, Karnataka, India \\ ${ }^{2}$ Manipal Hospitals, Department of Neurosurgery, Bangalore, Karnataka, India
}

\section{ABSTRACT}

\begin{abstract}
AIM: Micro-vascular anastomosis is a difficult art. It can be learnt and perfected only by practice using vessels of small animals in the laboratory. The purpose of the study was to practice various anastomotic techniques using rat femoral vessels and study the patency rates.
\end{abstract}

MATERIAL and METHODS: Sixty-four Sprague-Dawley rats were used in the study. The following procedures were conducted on rat femoral vessels; Femoral artery end-to-end anastomosis- classical method, one way up method, Femoral vein end-to-end anastomosis and end-to-side anastomosis. The animals were subsequently sacrificed at a later date to assess delayed patency.

RESULTS: The immediate patency rates for femoral artery end-to-end anastomosis- classical method ( $\mathrm{n}=10)$, one way up method $(n=16)$, femoral vein end-to-end anastomosis $(n=12)$ and end-to-side anastomosis $(n=26)$ were $100 \%, 81.3 \%, 58.3 \%$ and $53.8 \%$ respectively. The delayed patency rates (Mean follow-up=3.6 months) were $66.7 \%, 69.2 \%, 83.3 \%$ and $16 \%$ respectively. The clamp duration and suturing time significantly decreased and patency rates improved significantly in the latter half of the study.

CONCLUSION: The clamp duration, suturing time and patency rates improved considerably with time. The need of the hour is to establish well-planned training programs in centers with Animal Research facilities. This will promote younger colleagues and trainees to take up and perfect this difficult art. The present work could be used as a baseline study in this direction.

KEYWORDS: Microvascular anastomosis, Patency, Rat, Training

ABBREVIATIONS: STA-MCA: Superficial temporal artery- middle cerebral artery, STD: Standard deviation, USA: United States of America, MRCP: Microvascular Research Center Training Program

\section{INTRODUCTION}

$\mathrm{M}$ icro-vascular anastomosis is a difficult art. The technique of micro-vascular anastomosis can be learnt and perfected only by practice using vessels of small animals in the laboratory (21). Nevertheless, it has no endovascular correlate. It remains a crucial procedure in the treatment of multiple diseases. Indications include selected patients with ischemic cerebrovascular disease, particularly Moyamoya, complex aneurysms unamenable to direct clip reconstruction and tumors that may requiring vessel sacrifice to achieve complete resection $(9,18,19)$. Neurosurgical literature is lacking, in establishing a proper curriculum for training to learn and perfect this difficult art. Its importance though, has been emphasized from time to time; Professor M.Gazi Yaşargil, himself being a great proponent (21). 
The objective of the study was to practice various anastomotic techniques using rat femoral vessels and study the patency rates (immediate and delayed) in this animal model. The work was done as a baseline to establish a training curriculum in micro-vascular anastomosis; the successful completion of which should certify that the surgeon could do the similar surgeries in human patients.

\section{MATERIAL and METHODS}

The study was approved by the Institutional Animal Ethics Committee. Over a period of three years (2010-2013), sixtyfour Sprague-Dawley rats were used in the study. Using general anesthesia techniques, the following procedures were conducted on the rat femoral vessels:

Femoral artery end-to-end anastomosis- classical method (Group 1, n=10 rats), One way up method (Group 2, n=16 rats),

Femoral vein end-to-end anastomosis (Group 3, $n=12$ rats) and end-to-side anastomosis (vein-to-artery) was done using the epigastric vein in 23 rats; and femoral vein in 3 rats (Group 4).

All the surgeries were performed by the first author using an operating microscope. The animals were subsequently reexplored at a later date to assess delayed patency.

1. Femoral artery end-to-end anastomosis (Classical method) (Figure 1A-C): Using a groin incision, the femoral vessels were exposed. The femoral artery was dissected from its perivascular sheath. Usually, a branch on the underside of the artery had to be ligated and sacrificed. To relieve spasm, 1-2 cc of $1 \%$ lidocaine was applied. The artery was then clamped and divided. After removing the blood, the adventitia was thoroughly removed from the vessel ends. The vessel ends were subsequently dilated. A dark colored background was placed underneath to better visualise the vessel ends. The first two stay sutures were placed on the front wall, 120 degree apart. Then, two sutures (10-0 Ethilon $\AA$ ) were placed in between the first two sutures. The clamp (with the vessel) was turned over. The first suture (stay) on the back edges was placed exactly mid way between the two existing stay sutures. This divided the remaining unsutured part of the vessel's circumference into two equal halves. Usually, two sutures were paced in each of these halves. Hence, the anastomosis was completed using 9 sutures. The clamps were removed and gentle compression was applied for five minutes. The patency of the vessel was checked using empty-and-refill test. During the whole procedure, the vessel was held only by its adventitia. The whole thickness of the vessel wall was never grabbed with the forceps (1).

2. Femoral artery end-to-end anastomosis (One way up method): This is a useful technique of end-to-end anastomosis in tightly confined spaces. The initial steps of vessel ends' preparation were the same as in the classical method. The vessel suturing was completed without turning over the vessel. The first suture was placed as far away from yourself as possible. The next few sutures were placed on the back wall in an inverted fashion, starting closer to the first suture. Once the front wall was reached, regular sutures were placed (3).

3. Femoral vein end-to-end anastomosis (Classical method) (Figure 2A, B): Suturing veins is much more difficult as compared to the arteries. They are very fragile, difficult to dissect from the perivascular sheath, have very thin walls and have collapsible lumens. The suturing has to be done under saline to keep the lumen open. Due to slower flow, the amount of platelet thrombus that forms (at the site of any small insult or inaccuracy) is also greater. Barring these difficulties, the steps were the same as described in: Femoral artery end-toend anastomosis (Classical method) (4).
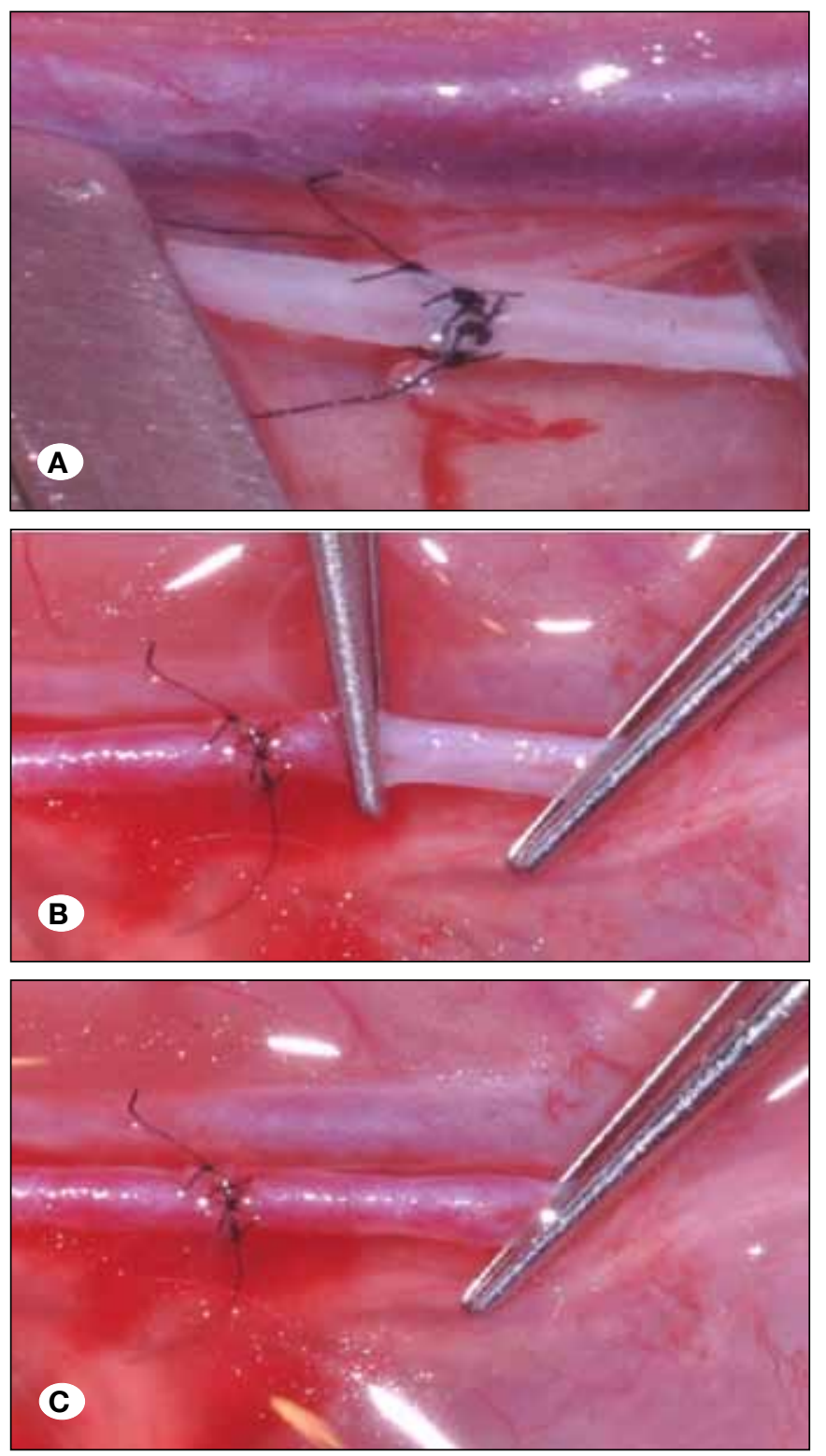

Figure 1: Femoral artery: End-to-end anastomosis has been completed. A) Just before removing the clamps. B) Empty and C) refill test in the distal segment to demonstrate good patency. 

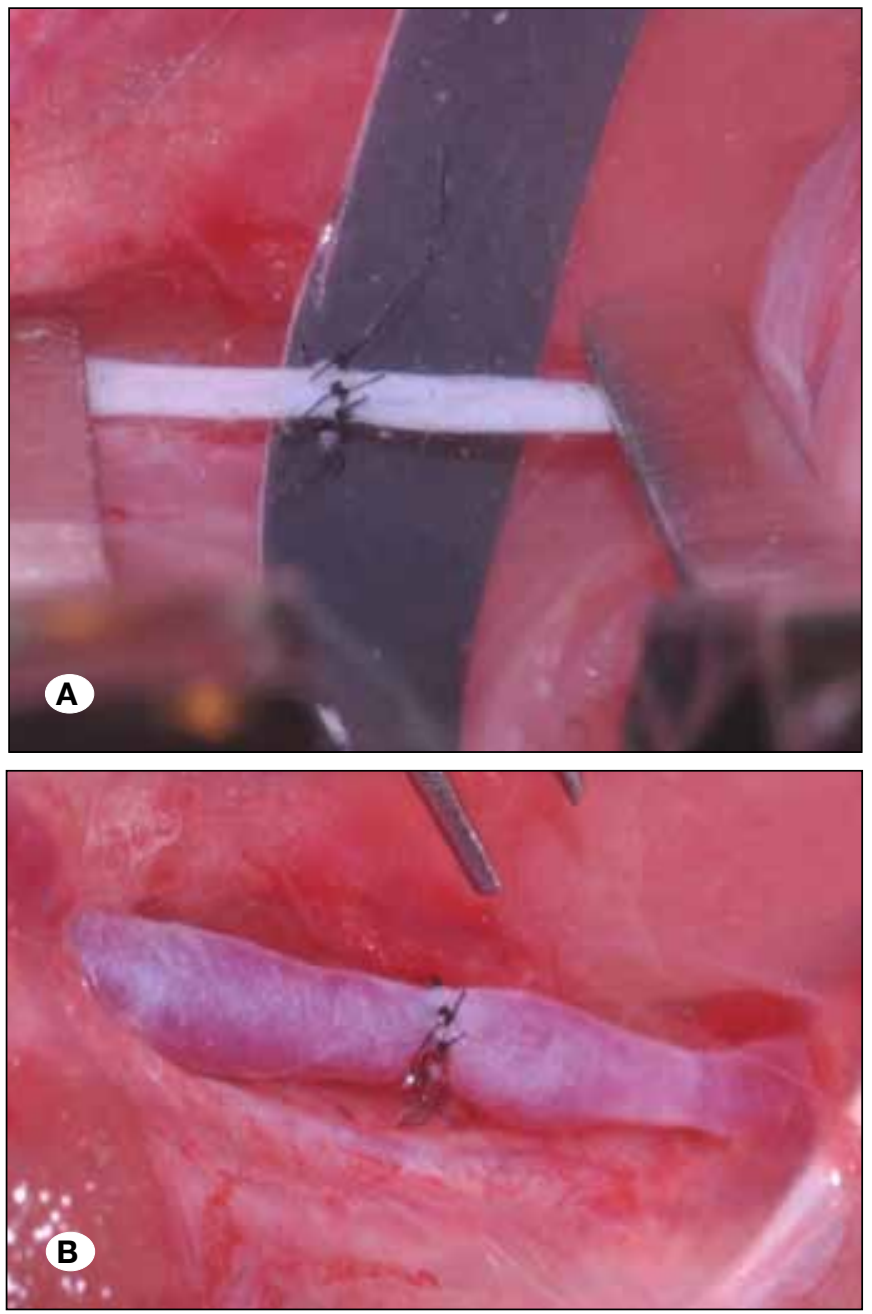

Figure 2: Femoral vein: End-to-end anastomosis has been completed. A) Before removing clamps and the colored background. B) Patent anastomosis after removing clamps and achieving hemostasis.
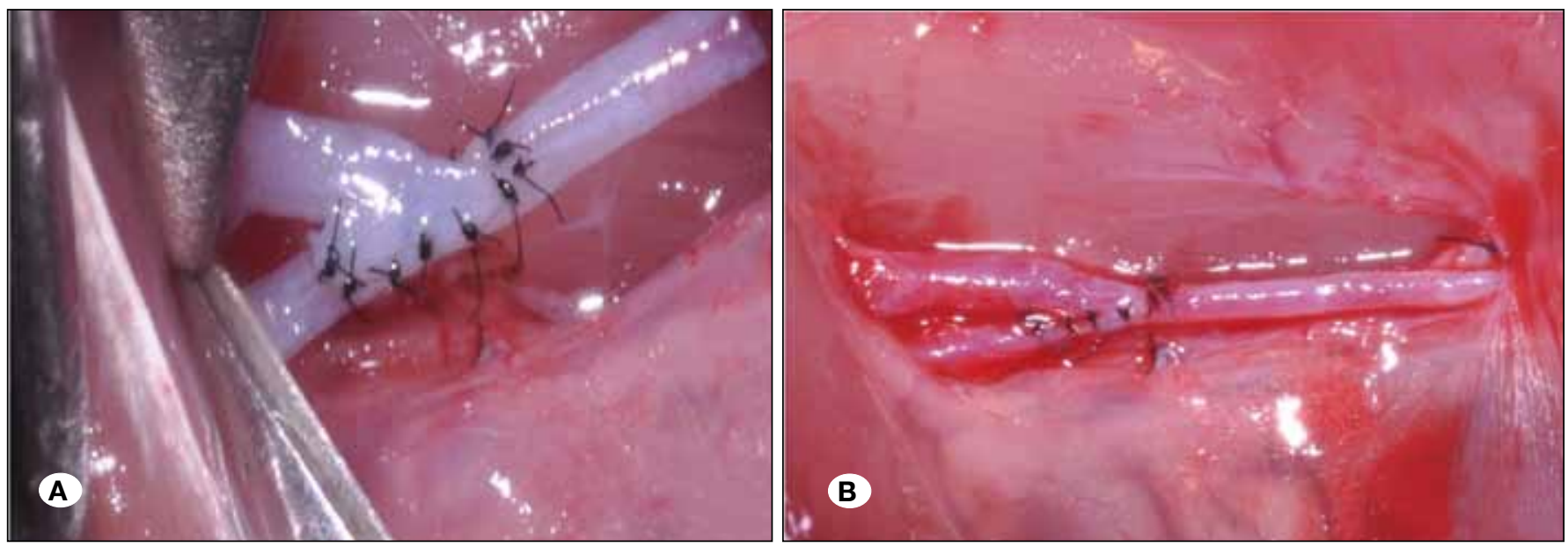

Figure 4: End-to-side anastomosis using femoral artery as recipient and femoral vein as the graft. A) After completion of anastomosis. B) After removal of clamps and achieving hemostasis. The femoral vein has become red.
4. Epigastric vein/Femoral vein-to-Femoral artery: End-toside anastomosis (Figures 3 and 4A, B): The initial steps of vessel preparation were the same as described in the classical method. In addition, epigastric vein (Figure 3) was dissected from its perivascular sheath, and mobilised towards the femoral artery. Fish-mouthing of the vein end was done in nine cases. After clamping the femoral artery, the adventitia was dissected from a segment of the vessel. A suture was passed transversely through the vessel wall, right where the middle of the arteriotomy will be. By lifting this suture, an elliptical opening was made in the arterial wall. The vein graft was the brought close to this opening. The heel and then the toe sutures were placed. The two walls were then sutured with interrupted sutures. The femoral vein was used instead of the epigastric vein in three cases (9).

After the procedure, the wound was closed. The animal was awakened and kept in postoperative room for 24 hours. especially those of hind limbs were observed. Under general

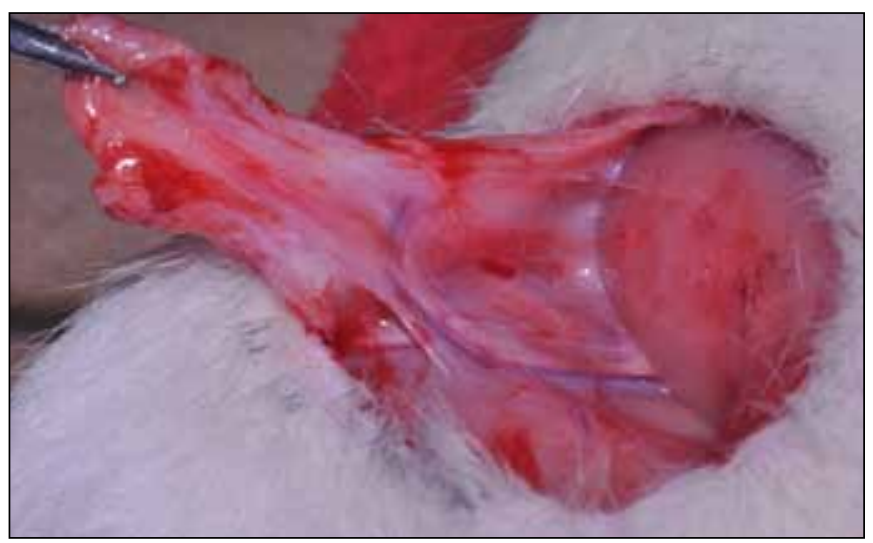

Figure 3: A low magnification view after giving the groin incision and mobilising the inguinal fat pad. The black arrow indicates the femoral vein; and the green arrow indicates the epigastric vein as it emerges from the fat pad. The rat was then shifted to its regular cage. Its movements 
anesthesia, at a later date, the wound was re-explored and delayed patency was checked by cutting the vein proximal and distal to the anastomoses. Color and pulsatility of both vessels were also noticed. The animals were subsequently sacrificed.

The parameters studied were clamping time, suturing time, immediate and delayed patency rates and the activities (especially leg movements) of the rat after the surgery.

\section{RESULTS}

After performing surgery in Groups 1,2,3 and 4 as previously described, both immediate and delayed patency rates were measured. The immediate patency rates were $100 \%, 81.3 \%$, $58.3 \%$ and $53.8 \%$ in Groups $1,2,3$ and 4 respectively. Irrespective of the anastomotic patency, the movements of the rat hind limbs were not affected by the surgery. At a mean follow-up period of 3.69 months (+/- 2.55), the animals were re-explored under general anesthesia and delayed patency was checked and found to be $66.7 \%, 69.2 \%, 83.3 \%$ and $16 \%$ in Groups 1,2,3 and 4 respectively.

\section{Clamp and Suturing duration (Table I)}

The clamp duration was defined as the time (in minutes) during which the vessel was clamped to do the anastomosis. The suturing duration was the time (in minutes) between inserting the first suture and removal of the clamp after completing the anastomosis. This was available for fifty-one rats. To see the effect of training and experience, all the groups were further divided into two halves. The clamp and suturing durations, along with the patency rates of the first half (initial surgeries) were compared to the second (later surgeries). In all the four groups, the clamp and suturing time decreased in the second (later) half of the study. This reduction was statistically significant $(p<0.05$, Mann Whitney test) for clamping duration in Groups 2 and 3; and for suturing duration in Groups 2 and 4. The patency rates improved in the Groups 2,3 and 4 in the second half. This improvement was statistically significant $(p<0.05$, Chi-square test) in Group 4.

\section{Group 4 (End-to-side anastomosis)}

Femoral vein was used as the donor vessel in 3 cases. All of them were patent immediately after surgery. However, the smaller caliber epigastric vein was used in the remaining 23 cases with an immediate patency rate of only $47.8 \%$ $(11 / 23)$. Fish mouthing technique was used in only 9 rats. The immediate patency rate in this group was $88.9 \%(8 / 9)$.

\section{DISCUSSION}

The femoral vessels of the rat provide several advantages for training. Their diameter is approximately $1 \mathrm{~mm}$, vein being slightly larger than the artery. They can be easily exposed by a groin incision, after mobilising the superficial fat pad. Clamping these vessels does not result in any significant injury to the rat leg, which was found to be functioning in all the experimental rats in the study, irrespective of the anastomosis patency.

Table I: Vessel Clamp Durations, Suturing Times and Immediate Patency Rates

\begin{tabular}{|c|c|c|c|}
\hline & $\begin{array}{l}\text { Clamp time (Mean +/- STD, } \\
\text { range in minutes) }\end{array}$ & $\begin{array}{l}\text { Suturing time (Mean +/- STD, } \\
\text { range in minutes) }\end{array}$ & Patency (Immediate) \\
\hline \multicolumn{4}{|c|}{ Group 1: Artery end-to-end: Classical method $(n=10)$} \\
\hline First five & $57+/-16.54(33-78)$ & $31.67+/-5.77(25-35, n=3)$ & $100 \%$ \\
\hline Last five & $42.8+/-8.53(33-50)$ & $30.80+/-4.60(25-37, n=5)$ & $100 \%$ \\
\hline$\underline{p \text { value }}$ & 0.094 Mann-Whitney Test & 0.763 Mann-Whitney Test & \\
\hline \multicolumn{4}{|c|}{ Group 2: Artery end-to-end: One-way up method $(n=16)$} \\
\hline First eight & $67.12+/-16.94(50-97)$ & $65.25+/-16.28(45-82, n=4)$ & $75 \%$ \\
\hline Last eight & $40.62+/-8.42(30-54)$ & $29.38+/-7.54(20-41, n=8)$ & $87.5 \%$ \\
\hline $\mathrm{p}$ value & 0.002 Mann-Whitney Test & 0.006 Mann-Whitney Test & 0.522 Chi-Square Test \\
\hline \multicolumn{4}{|c|}{ Group 3: Vein end-to-end $(n=12)$} \\
\hline First six & $88.00+/-26.65(50-130)$ & & $50 \%$ \\
\hline Last six & $56.33+/-19.90(35-92)$ & $38.40+/-9.66(30-55, n=5)$ & $66.7 \%$ \\
\hline $\mathrm{p}$ value & 0.037 Mann-Whitney Test & & 0.558 Chi-Square Test \\
\hline \multicolumn{4}{|c|}{ Group 4: End-to-side $(n=26)$} \\
\hline First thirteen & $84.23+/-19.03(57-119)$ & $64.85+/-16.39(39-85, n=13)$ & $23.1 \%$ \\
\hline Last thirteen & $71.30+/-10.93(55-93)$ & $50.30+/-8.45(35-64, n=13)$ & $84.6 \%$ \\
\hline $\mathrm{p}$ value & 0.0950 Mann-Whitney Test & 0.025 Mann-Whitney Test & 0.002 Chi-Square Test \\
\hline
\end{tabular}


The re-exploration surgery (to check delayed patency) is not cumbersome (1-4).

Suturing veins is much more difficult as compared to the arteries. Reasons have been mentioned in the methodology. It was interesting to note that unlike other groups, the delayed patency rates of venous end-to-end anastomosis group was more as compared to the immediate patency rates $(83.3 \% \mathrm{Vs}$ $58.3 \%$ ). Hui et al.(11) performed 300 standard microsurgical anastomoses on rat femoral veins. The patency of each anastomosis was assessed at regular intervals within a 2 week period. The results showed that recanalization was frequently observed in venous anastomosis. This could explain our observation. They concluded that the optimal time to assess outcome of venous patency was 1 to 2 days after repair and not immediately after surgery (as done in the current study). Hui et al. (12), in another study, tried to see the role of experience in doing these difficult venous anastomosis surgeries. They concluded that, even in experienced hands, the best achievable patency rate was $88 \%$.

What is the best time to study delayed patency? In the experimental studies, performed by plastic and reconstructive surgeons, this period has ranged from 3 to 14 days $(5-7,11,17)$. Harashina et al., using scanning electron microscope, showed that after 5 days of surgery, the micro-anastomosis area is covered with a pseudo-intima. The regeneration of the intima is completed within two weeks (10). Hence, two weeks appears to be an optimum time to study the delayed patency. In the present study, the mean duration from surgery to sacrifice (to study delayed patency) was 3.69 months. This could have resulted in lower delayed patency rates, and can be considered as a drawback of the study. Newer modalities like intraoperative near-infrared indocyanine-green (ICG) angiography and mini Doppler systems have been used to evaluate anastomosis patency in the rat femoral vessels $(16,20)$. Though expensive, these techniques could avoid a reexploration surgery to check for delayed patency.

End-to-side is the most commonly done anastomosis in neurosurgical practice. We used a vein to artery model in all the experiments $(n=26)$. The smaller epigastric vein was used in 23 cases and the larger femoral vein in three of them. The patency rates (Immediate 53.8\%, Delayed 16\%) were the least satisfactory in this experimental group. The technique of fish mouthing of the vessel end was used in only 9 cases, with an immediate patency rate of $88.9 \%(8 / 9)$. The causes of failure of an end-to-side anastomosis can be technical and flow related. Trying to learn from our experience, currently, we are doing these experiments using the femoral artery to vein model. In our opinion, this mimics the "STA-MCA anastomosis" surgery more closely. Fish mouthing of the distal end of the femoral artery is being practised in all experiments now.

The principles of microsurgery are not unique to the field of neurosurgery. Microvascular surgery has been practised by plastic and reconstruction surgeons since many decades $(10,15)$. Lack of a comprehensive standardized microsurgery training program, is a hindrance in the training of several trainees who are very enthusiastic about learning it. To address this need, Komatsu et al. established the Microvascular Research Center Training Program (MRCP) in 2005 (14), comprising progressive skill level requirements to help trainee surgeons (Plastic and Reconstructive surgery) acquire microsurgical skills.

The MRCP comprises five stages of training, each with specific passing requirements. Stages 1 and 2 involve anastomosing silicone tubes and blood vessels of chicken carcasses, respectively, within 20 minutes. Stage 3 involves anastomosing the femoral artery and vein of live rats with a 1 -day patency rate of $>80 \%$. Stage 4 requires replantation of free superficial inferior epigastric artery flaps in rats with a 7 -day success rate of $>80 \%$. Stage 5 involves successful completion of one case of rat replantation/transplantation. Trainee surgeons who participated in this course were not permitted to anastomose human blood vessels until they passed stage 4 . The duration of the course was 3 months.

Stage 4 and 5 of MRCP are probably not relevant for the training of a neurovascular surgeon. The authors do not give details of the surgeries performed in Stage 3, in order to achieve a 1 day patency rate of $>80 \%$. The importance of improvement in patency rates and decrease in suturing times with training, consistent with the present study is highlighted. The need of the hour (for various Neurosurgical Associations across the world) is to establish similar training programs to promote younger colleagues and trainees; in order to learn this difficult art. The duration of the training program, types of surgeries, animal models, cut off patency rates and suturing times need to be discussed in detail. The present work could be used for a baseline study in this direction.

Dr.M.Gazi Yaşargil spent nearly three years (1964-1967) working in the laboratory of Dr. R.M. Peardon Donaghy in Burlington, Vermont, USA. During this time, he performed various experiments on blood vessels of rabbits and dogs $(8,13)$. It was here that he conceptualised the surgery of STA-MCA anastomosis. In one of his articles (21), he wrote "The quintessence of my 33-year long mission has been to convince my neurosurgical colleagues of the absolute of necessity laboratory training in micro-techniques before their application to humans in the operating room."

\section{CONCLUSION}

Training in the art of microvascular anastomosis is difficult and can be learnt only by practising on animal models. The patency rates and suturing duration improve considerably with training. Suturing veins is more difficult than the arteries. The need of the hour is to establish well-planned training programs in centers with Animal Research facilities. This will promote younger colleagues and trainees to take up and perfect this difficult art; and certify them for doing similar surgeries in human patients. The present study can serve as a baseline in this regard.

\section{ACKNOWLEDGEMENTS}

The authors would like acknowledge the support of the entire staff at Central Animal Research Facility, NIMHANS, Bangalore, India especially Mr.Ramachandran R for this work. We are also thankful to the Department of Mental Health Education, NIMHANS, Bangalore, India for their camera work. 


\section{REFERENCES}

1. Acland RD, Sabapathy SR: Arterial anastomoses: Common femoral artery. Acland's Practice Manual for Microvascular Surgery. $3^{\text {rd }}$ ed. Indian Society for Surgery of the Hand, 2008:40-60

2. Acland RD, Sabapathy SR: End to side anastomosis: Common femoral artery. Acland's Practice Manual for Microvascular Surgery. $3^{\text {rd }}$ ed. Indian Society for Surgery of the Hand, 2008: 88-97

3. Acland RD, Sabapathy SR: One way up anastomosis. Acland's Practice Manual for Microvascular Surgery. $3^{\text {rd }}$ ed. Indian Society for Surgery of the Hand, 2008: 81-83

4. Acland RD, Sabapathy SR: Venous anastomosis: common femoral vein. Acland's Practice Manual for Microvascular Surgery. $3^{\text {rd }}$ ed. Indian Society for Surgery of the Hand, 2008: 61-68

5. Adams WP Jr, Ansari MS, Hay MT, Tan J, Robinson JB Jr, Friedman RM, Rohrich RJ: Patency of different arterial and venous end-to-side microanastomosis techniques in a rat model. Plast Reconstr Surg 105:156-161, 2000

6. Aygun $\mathrm{H}$, Yildirim OS: Vertical mattress suture technique: An alternative vascular anastomosis. J Reconstr Microsurg 24(6):397-404, 2008

7. Decherd ME, Calhoun $\mathrm{KH}$ : The effect of tension on patency of rat femoral artery anastomoses. Arch Facial Plast Surg 5:8385, 2003

8. Donaghy RMP, Yasargil MG (eds): Micro-vascular Surgery Report of First Conference, October 6-7, 1966, Burlington, Vermont. St.Louis, Mosby, 1967

9. Guzman R, Lee M, Achrol A, Bell-Stephens T, Kelly M, Do HM, Marks MP, Steinberg G: Clinical outcome after 450 revascularization procedures for Moyamoya disease. J Neurosurg 111:927-935, 2009

10. Harashina T, Fujino T, Watanabe S: The intimal healing of microvascular anastomoses. Plast Reconstr Surg 58:608-613, 1976

11. Hui KC, Zhang F, Shaw WW, Kryger Z, Piccolo NS, Harper A, Lineaweaver WC: Learning curve of microvascular venous anastomosis: A never ending struggle? Microsurgery 20:2224,2000
12. Hui KC, Zhang F, Shaw WW, Taylor A, Komorowska-Timek E, Lineaweaver WC: Assessment of the patency of microvascular venous anastomosis. J Reconstr Microsurg 18:111-114, 2002

13. Jacobson JH II, Wallman LJ, Schumacher GA, Flanagan M, Suarez EL, Donaghy RPM: Microsurgery as an aid to middle cerebral artery endarterectomy. J Neurosurg 19:108-115, 1962

14. Komatsu S, Yamada K, Yamashita S, Sugiyama N, Tokuyama E, Matsumoto K, Takara A, Kimata Y: Evaluation of the microvascular research center training program for assessing microsurgical skills in trainee surgeons. Arch Plast Surg 40:214-219, 2013

15. Lauritzen C, Bagge U: A technical and biomechanical comparison between two types of microvascular anastomoses. An experimental study in rats. Scand J Plast Reconstr Surg 13:417-421, 1979

16. Mücke T, Wolff KD, Wagenpfeil S, Hölzle F, Scholz M: Reliability of near-infrared angiography and micro-Doppler sonography for evaluating microvascular anastomoses. Plast Reconstr Surg126:1506-1514, 2010

17. Rickard RF, Engelbrecht GH, Hudson DA: Experimental investigation of two techniques of arterial microanastomosis used to manage a small-to-large diameter discrepancy. J Plast Reconstr Aesthet Surg 64:1088-1095, 2011

18. Scott RM, Smith ER: Moyamoya disease and Moyamoya syndrome. N Engl J Med 360:1226-1237, 2009

19. Sekhar LN, Natarajan SK, Ellenbogen RG, Ghodke B: Cerebral revascularization for ischemia, aneurysms, and cranial base tumors. Neurosurgery 62 Suppl 3:1373-1408, 2008

20. Uraloglu M, Orbay H, Livaoglu M, Aydin N, Sarac N, Alagoz S: An objective evaluation of an injured vessel wall using fluorescein sodium before microvascular anastomosis in an experimental rat model. J Reconstr Microsurg 27:321-326, 2011

21. Yasargil MG: A legacy of microneurosurgery: Memoirs, lessons and axioms. Neurosurgery 45:1025-1092, 1999 